Article

\title{
Preparation and Characterization of Directionally Freeze-cast Copper Foams
}

\author{
Aurelia I. Cuba Ramos ${ }^{1,2}$ and David C. Dunand ${ }^{1, *}$
}

1 Department of Material Science and Engineering, Northwestern University, Evanston, IL 60208, USA; E-Mail: a.cubaramos@googlemail.com

2 Institute for Applied Materials, Karlsruhe Institute of Technology, Karlsruhe 76131, Germany

* Author to whom correspondence should be addressed; E-Mail: dunand@northwestern.edu; Tel.: +1-847-491-5370.

Received: 2 June 2012; in revised form: 9 July 2012 / Accepted: 30 July 2012/

Published: 9 August 2012

\begin{abstract}
Because of their excellent thermal and electric conductivities, copper foams are ideally suited for applications such as heat exchangers, catalyst supports and EMI-shields. Here, we demonstrate the preparation of copper with $\sim 80 \%$ aligned, elongated, interconnected pores via directional freeze casting, a well established processing technique for porous ceramics. First, an aqueous slurry of 40-80 nm cupric oxide powders was directionally solidified, resulting in a preform consisting of elongated, aligned dendrites of pure ice separated by interdendritic ice walls with high oxide powder content. Oxide rather than metallic nanometric particles are used, as the latter would oxidize rapidly and uncontrollably when suspended in the aqueous solution used during directional casting. The preforms were then freeze-dried to sublimate the ice and sintered in a hydrogen-bearing atmosphere to reduce the copper oxide to metallic copper particles and densify these copper particles. Microstructural analysis of the copper foams shows that three types of porosities are present: (i) aligned, elongated pores replicating the ice dendrites created during the freeze-casting process; (ii) micro-porosity in the partially sintered copper walls separating the elongated pores; and (iii) cracks in these copper walls, probably created because of shrinkage associated with the reduction of the oxide powders.
\end{abstract}

Keywords: porous materials; metallic foams; copper; copper oxide; freeze-casting; chemical reduction; sintering; oriented pores 


\section{Introduction}

Copper foams, due to the excellent thermal and electric conductivities of the copper matrix, are ideally suited for a wide range of industrial applications, such as heat exchangers, catalyst supports and EMI-shielding, while the ductility of copper leads to good foam mechanical energy absorption in compression.

Research in recent years has been directed towards developing new methods and optimizing existing processing techniques to create copper foams. Typical processing routes to produce copper foams include space-holder techniques, dissolution of a less noble element in binary copper alloys, investment casting and melt gas injection [1-10].

The present work aims to create copper foams via freeze casting [11-13]. This processing technique consists of freezing an aqueous suspension of powders such that pure ice dendrites push the powders in interdendritic space, sublimation of the ice, and sintering of the resulting green body, where pores replicate the dendrites. This method is well established for producing porous ceramics but had never been applied to metals, until recently when Chino and Dunand and Li and Dunand [14,15] used it to create titanium with elongated pores, starting from titanium powders.

In comparison with the established processing techniques for copper foams mentioned above, directional freeze casting as a processing route for creating copper foams offers the opportunity to create aligned, elongated pores, using space holders (ice) which are created in situ during solidification. This method further offers the ability to manipulate the pore fraction, diameter, spacing and orientation.

Here, we demonstrate for the first time that directional freeze casting can be used to create copper foams with aligned, elongated pores, using copper oxide powders suspended in water which are subsequently sintered in a hydrogen atmosphere to simultaneously reduce the copper oxide to pure copper and densify the copper particles. Oxide rather than metallic particles are selected here to avoid uncontrolled oxidation in aqueous solutions, and reduce the tendency for powder settling. The resulting microstructure of the copper foam is analyzed, and some factors affecting the formation of the porous structure are further investigated.

\section{Results and Discussion}

\subsection{Foam Microstructure}

Optical micrographs of longitudinal cross-sections of a copper foam obtained from a 10 vol \% cupric oxide $(\mathrm{CuO})$ suspension (sample 1) and from a 15 vol \% $\mathrm{CuO}$ suspension (sample 2) are depicted in Figure $1 \mathrm{a}-\mathrm{c}$ and Figure $2 \mathrm{a}-\mathrm{c}$, respectively. The micrographs show a high amount of porosity in the foams, which is highly aligned in some regions of the samples.

The longitudinal cross-sections at low magnification in Figures 1a and 2a show that, in up to about one third of the total height of the sample 1, the copper walls are aligned in the freezing direction, followed by a zone where the structure appears to be more isotropic and the pores are much smaller. For sample 2, elongated pores start at the bottom of the sample and extend up to about $90 \%$ of the total height of the sample, followed by a thin layer where solidification was non-directional. The optical analysis indicates that significant particle settling occurred during solidification of the 10 vol \% slurry, 
causing a gradient in solids content and consequently in the density of the freeze-cast structure. By contrast, an initial $\mathrm{CuO}$ powder loading of $15 \mathrm{vol} \%$ in the slurry is sufficient to create ice dendrites over most of the sample height, and particle pushing in the interdendritic space indicates that electrostatic stabilization of the powders by adjusting the $\mathrm{pH}$ value to 10 through addition of $\mathrm{KOH}$ is successful. The optical micrographs at higher magnifications (Figure 1b, c and Figure 2b, c) reveal the structure of the copper walls which consist of sintered copper particles and micro-pores within the walls. Cracks and some larger pores are visible within the walls as well. These cracks were not observed in the microstructure of the directionally freeze-cast titanium foams prepared by sintering of metallic Ti powders by Chino and Dunand and by Li and Dunand [14,15]. One possible explanation might be that these cracks form during the reduction of the cupric oxide, which involves a volume decrease due to the higher density of copper compared to cupric oxide.

Three populations of pores can be distinguished: aligned, elongated pores; cracks in the copper walls and micro-porosity within the copper walls. In addition, Figure $2 \mathrm{~d}$ and $2 \mathrm{e}$ show optical micrographs of transverse cross-sections of the sample 2. There are several regions were pores are aligned. These regions replicate colonies of parallel ice dendrites, as also observed in titanium foams [14,15].

The average pore diameter for sample 1 and sample 2 is $75 \pm 36 \mu \mathrm{m}$ and $91 \pm 33 \mu \mathrm{m}$, respectively. It should be noted that the average pore diameter for sample 1 has been measured only for the area where directional solidification occurred. Archimedes measurements revealed a total porosity of $\sim 80 \%$ and were verified by means of the software ImageJ. The relative density of $\sim 20 \%$ is higher than the loading fraction of $15 \%$ as expected from densification during sintering.

Figure 1. Optical micrographs of sample 1 obtained from a $10 \mathrm{vol} \% \mathrm{CuO}$ suspension. Longitudinal cross-sections at (a) low, (b) medium and (c) high magnification. Copper is reddish and pores are filled with black resin.

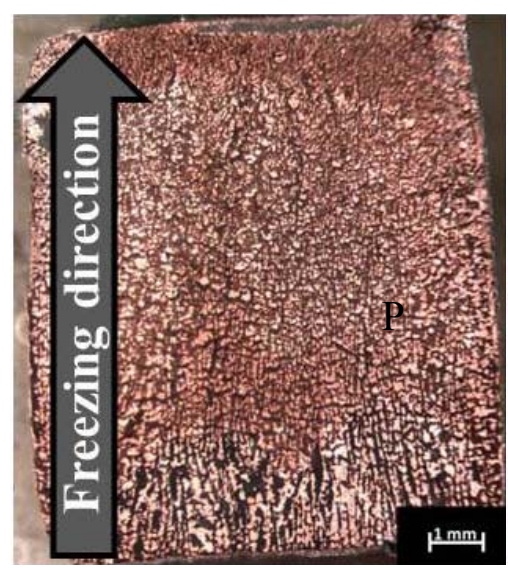

(a)

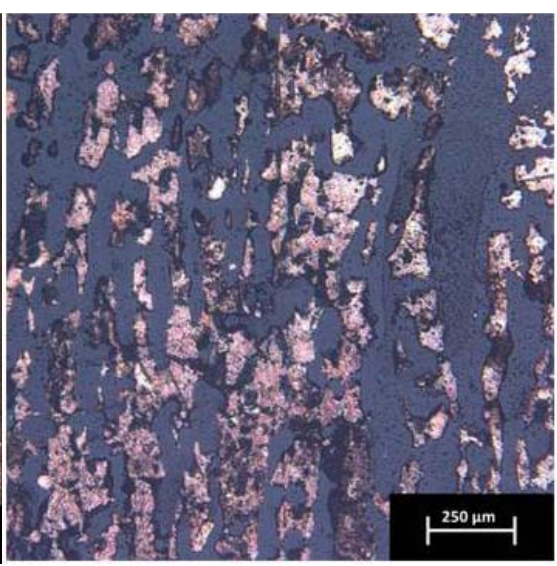

(b)

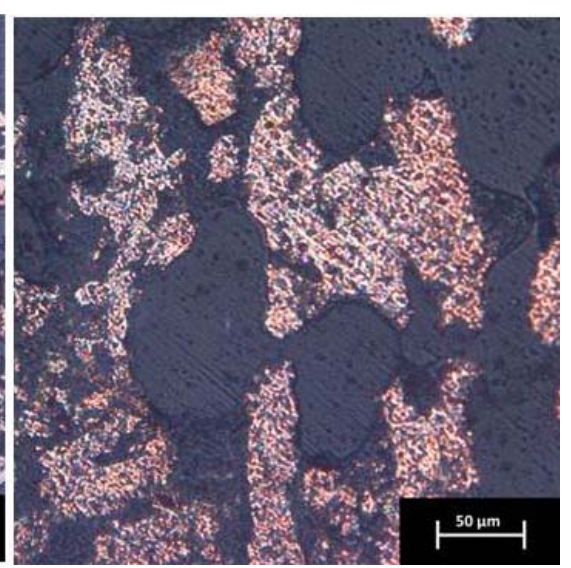

(c) 
Figure 2. Optical micrographs of sample 2 obtained from a $15 \mathrm{vol} \% \mathrm{CuO}$ suspension. Longitudinal cross-sections, at (a) low, (b) medium and (c) high magnification. Transverse cross-sections at (d) medium and (e) high magnification.

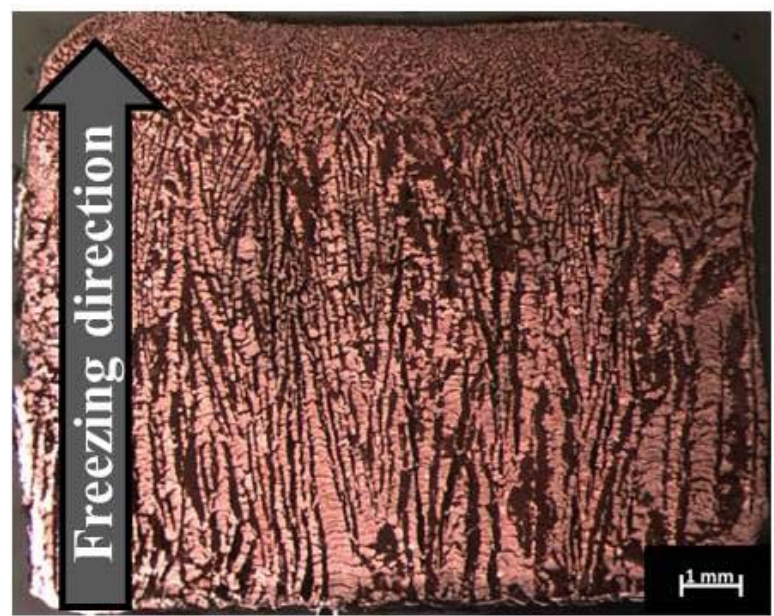

(a)

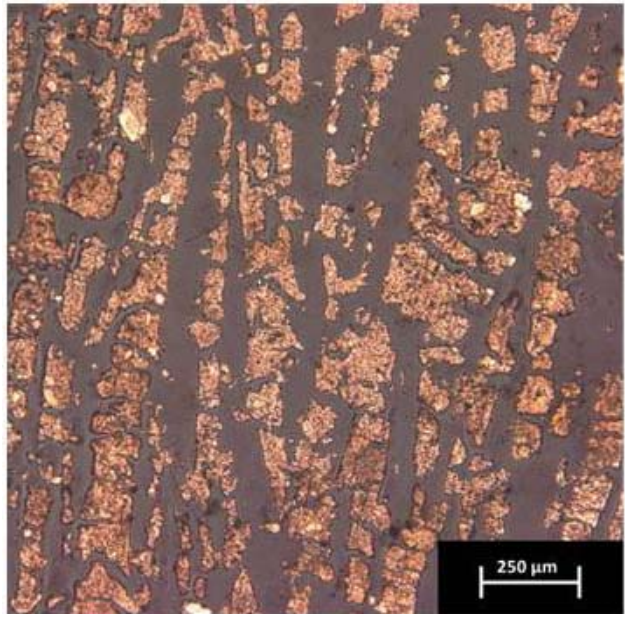

(b)

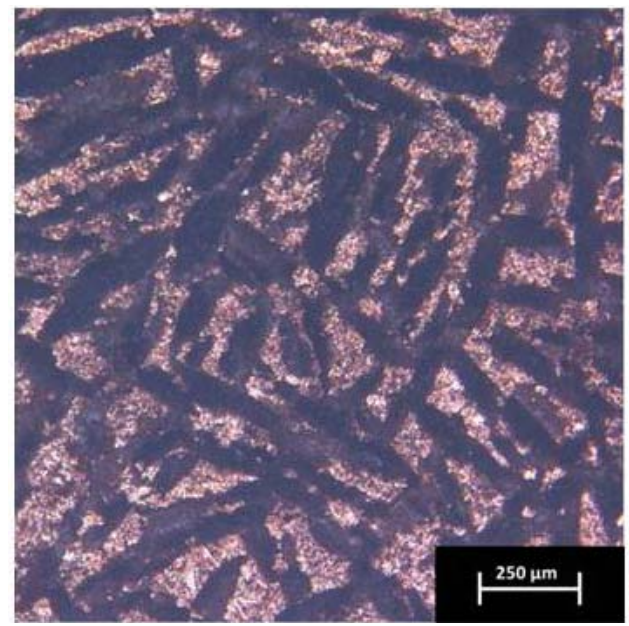

(d)

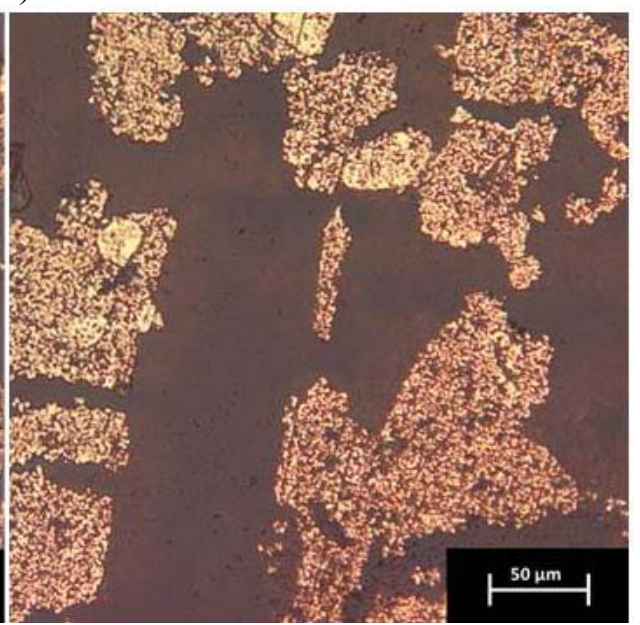

(c)

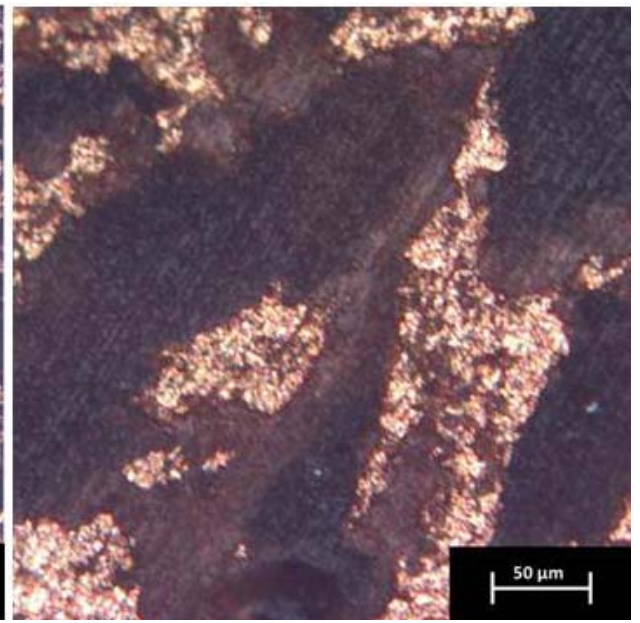

(e) 


\subsection{Influence of Sintering Parameters}

Through control of the temperature gradient and powder characteristics, it has been shown that the pore fraction, diameter, spacing and orientation could be manipulated in titanium foams created by a similar freeze-casting method $[14,15]$. It is expected that the pore characteristics of the present copper foam can be similarly controlled.

Figure 3 shows the effect of additional sintering upon the microstructure of the copper foam walls. The figure depicts two pieces of the same foam obtained from a $15 \mathrm{vol} \% \mathrm{CuO}$ slurry, from which the second piece (Figure $3 \mathrm{~b}$ ) was re-sintered for $4 \mathrm{~h}$ at $800{ }^{\circ} \mathrm{C}$. It is evident that micro-porosity was significantly reduced after the re-sintering step. This densification is expected due to the high homologous temperature at which small pores sinter by flux of vacancies.

Figure 3. Optical micrographs of longitudinal cross-sections of $\mathrm{Cu}$ foams (a) as sintered $\left(2 \mathrm{~h} / 600{ }^{\circ} \mathrm{C}\right)$; (b) after second sintering $\left(4 \mathrm{~h} / 800^{\circ} \mathrm{C}\right)$.

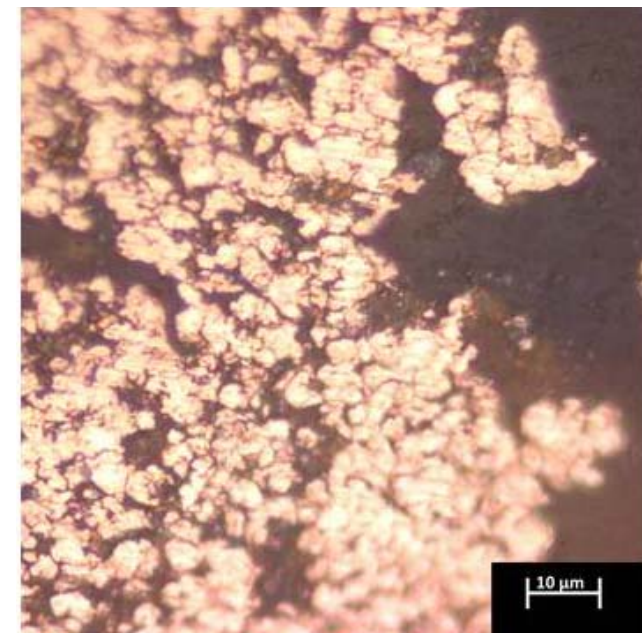

(a)

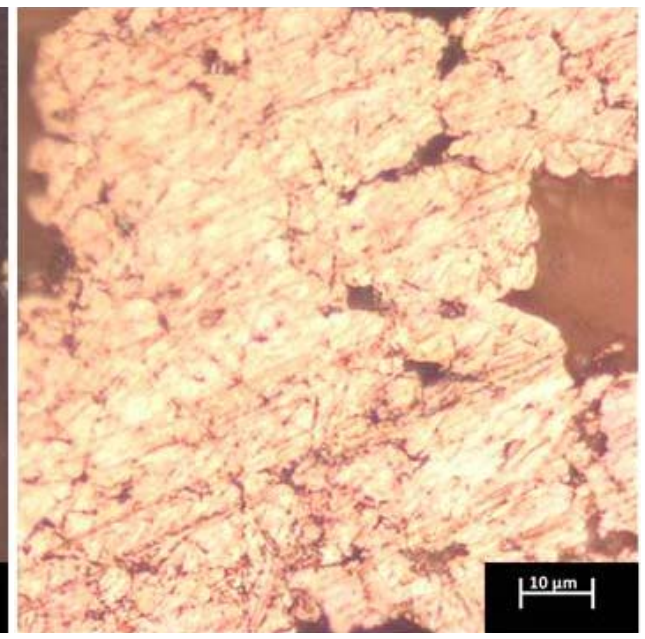

(b)

\section{Experimental Procedures}

\subsection{Slurry Preparation}

Powders of cupric oxide $\left(6.31 \mathrm{~g} \cdot \mathrm{cm}^{-3}\right.$ density) with an average particle size of $40-80 \mathrm{~nm}$ and with 99.9\% purity were purchased from Inframat Advanced Materials (Manchester, CT, USA). For all experiments, 0.2 wt \% agar binder (Eden Foods Inc., Clinton, MI, USA) was stirred in deionized water. The mixture was then boiled to dissolve the agar and degas the water and subsequently allowed to cool down. The slurries for freeze casting were prepared in a polypropylene test tube with $13 \mathrm{~mm}$ inner diameter and $1.2 \mathrm{~mm}$ average wall thickness. To improve heat conduction and promote unidirectional freezing, the conical bottom of the test tubes was sectioned and replaced by a copper cylinder of $12.7 \mathrm{~mm}$ diameter and $\sim 1 \mathrm{~cm}$ height that was glued in place with silicone.

For the first set of experiments, $2.1 \mathrm{~g}$ of cupric oxide powders and $1 \mu \mathrm{L}$ of neutral detergent as a dispersant were stirred in $3.0 \mathrm{~mL}$ of the prepared water/agar mixture. The resulting slurry contained 10 vol \% of copper oxide powders and was subsequently degassed in a vacuum desiccator. The slurry 
was cooled to a few degrees above the freezing point of water and was then stirred for about $30 \mathrm{~s}$ to suspend the powders homogeneously before freeze-casting.

In the second set of freeze-cast experiments, aqueous suspensions containing $15 \mathrm{vol} \% \mathrm{CuO}$ were prepared. In order to increase the amount of solids content from 10 to $15 \mathrm{vol} \%$, it became necessary to reduce particle-particle attraction and to improve the stability of the suspension. Ceramic suspensions can be stabilized in both basic and acid electrolytes. Initial tests, where hydrochloric acid ( $\mathrm{HCl})$ and potassium hydroxide $(\mathrm{KOH})$ were added to decrease or increase the $\mathrm{pH}$ value of the suspension, respectively, revealed that stabilization in the basic range was easier to achieve. Therefore, sedimentation experiments in the basic range were conducted. For this purpose, six different dilute suspensions were prepared. In each case, $6 \mathrm{~mL}$ of deionized water and $2 \mathrm{~g}$ of $\mathrm{CuO}$ powders were used. The water was filled into small cylindrical glass beakers and $\mathrm{KOH}$ was added to five of the six testing solutions to vary the $\mathrm{pH}$ values from 8 to $9,10,11$ and 12. Powders were stirred in and the suspensions were sonicated for $30 \mathrm{~min}$ to break down large agglomerates. When stirring in the powders, it was observed, that suspensions of $\mathrm{pH} 10$ and 11 were less viscous, and powders dispersed more easily. Finally, $2 \mu \mathrm{L}$ of neutral detergent were added for steric stabilization, and suspensions were stirred one more time. The dispersant was added after sonicating, to avoid foaming of the slurries. The suspensions were allowed to stand for $3 \mathrm{~h}$. The height of the sedimentation layer was subsequently measured for the individual suspensions. Comparison of the results showed that the height of the sedimentation layer was minimal for the suspensions with $\mathrm{pH} 10$ and 11 . This indicates that these suspensions are well deflocculated, because they settled relatively slowly and the sediment was relatively dense. Suspensions of $\mathrm{pH}$ 7, 8, 9 and 12 appeared more coagulated, since they settled rapidly and the sediment was of relatively low density. These results are in good agreement with the observations made when stirring the powders in the water.

Consequently, in the second set of freeze-cast experiments, the $\mathrm{pH}$ value of the water/agar mixture was adjusted to $\mathrm{pH} 10$ by adding $\mathrm{KOH}$. Afterwards, $2.0 \mathrm{~g}$ of cupric oxide powders were stirred in $3.0 \mathrm{~mL}$ of the prepared water/agar/KOH mixture and sonicated for $30 \mathrm{~min}$. Thereafter, $1.34 \mathrm{~g}$ of cupric oxide powders were stirred in the slurry and the resulting 15 vol \% slurry was sonicated again for $30 \mathrm{~min}$. Finally, $1 \mu \mathrm{L}$ of neutral detergent was added and the slurry was degassed in a vacuum desiccator. As in the first set of experiments, the slurry was cooled to a few degrees above the freezing point of water and was then stirred for about $30 \mathrm{~s}$ to suspend the powders homogeneously before freeze-casting.

\subsection{Freeze Casting and Sintering}

The setup designed for freezing the aqueous slurries consisted of a secondary container filled with a sodium chloride $(\mathrm{NaCl})$ /ice/water cooling bath mixture and is illustrated in Figure 4. The initial temperature of the cooling bath varied in the range of $-14{ }^{\circ} \mathrm{C}$ to $-19{ }^{\circ} \mathrm{C}$ and stayed almost constant during the whole freezing stage. The sides and the bottom of the secondary container were insulated with polystyrene foam to delay the rise in temperature of the cooling bath mixture. A small copper rod was attached to the bottom center of the secondary container and served as a conductor between the cooling bath and the test tube containing the slurry. The bottom of the test tubes used, i.e., the copper cylinder, and the copper rod had identical diameters, so that the test tube could be placed on top of the 
copper rod. To realize directional solidification of the slurry, the sides and top of the test tube were insulated with polystyrene foam. The test tube was removed from the freezing unit after $1.5 \mathrm{~h}$.

The frozen ice/CuO billets were then sublimated for $24 \mathrm{~h}$ in a lyophilizer under a $0.060 \mathrm{mbar}$ vacuum at $-83{ }^{\circ} \mathrm{C}$. For the sintering of the resulting ice-free green bodies, a tube furnace was used with a flowing atmosphere of $\mathrm{Ar}-4 \% \mathrm{H}_{2}$ to reduce the cupric oxide to metallic copper. The sample was heated at a constant heating rate of $10{ }^{\circ} \mathrm{C} \mathrm{min}-1$ until it reached $600{ }^{\circ} \mathrm{C}$. The holding time was $2 \mathrm{~h}$ and the sample was then furnace-cooled.

Figure 4. Schematic of freezing setup.

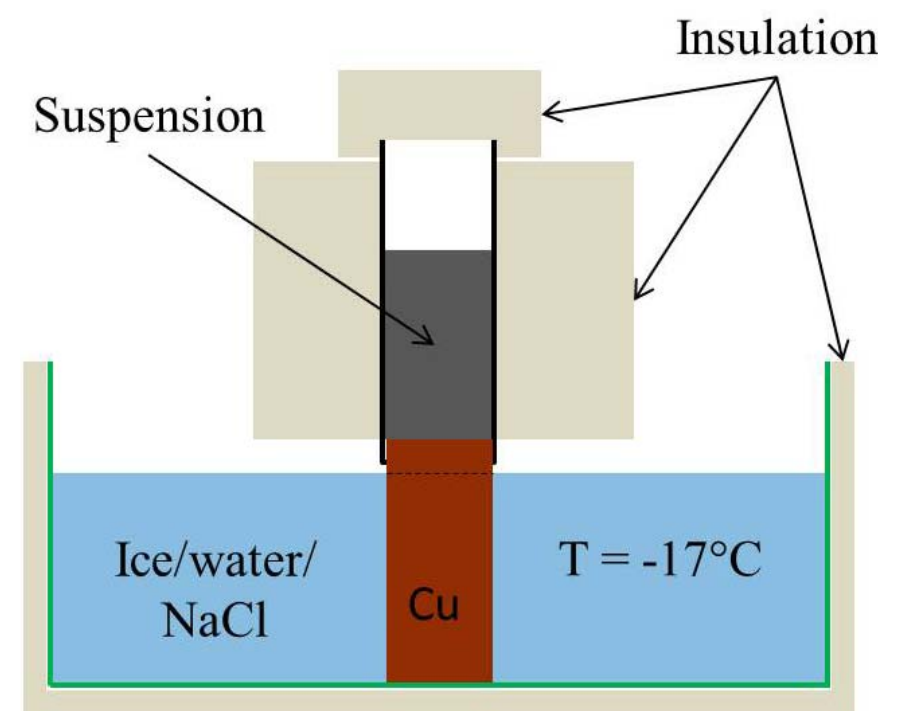

One additional experiment was conducted, in which a sintered billet was broken in two pieces. One piece was re-sintered with a heating rate of $10{ }^{\circ} \mathrm{C} \mathrm{min}{ }^{-1}$ and holding time of $4 \mathrm{~h}$ at $800{ }^{\circ} \mathrm{C}$, to investigate the influence of further sintering on the foam microstructure.

\subsection{Microstructural Analysis}

The freeze-cast and sintered billets were cut such that cross-sections parallel and perpendicular to the pore longitudinal direction could be polished and imaged. The width of twenty pores was measured on an image of the transverse cross-section using the Image J software.

Porosity was measured by the Archimedes method, where the samples were weighted in air and in water (after filling pores with grease in the latter case). These results were verified by means of the ImageJ software, where porosity was calculated from the digitally colored optical micrographs of the epoxy-filled sample by measuring the area occupied by the epoxy.

\section{Conclusions}

Copper foams with aligned, elongated macro-pores were created by directional freeze casting of aqueous slurries of cupric oxide powders followed by freeze-drying to remove ice dendrites and annealing in an $\mathrm{Ar}-4 \% \mathrm{H}_{2}$ atmosphere to reduce the cupric oxide to copper and to sinter the powders. To achieve formation of aligned, elongated pores, sufficient solids loading and stability of the suspension during the freezing stage are required. Cupric oxide nanoparticle suspensions can be 
successfully stabilized by adjusting the $\mathrm{pH}$ of the suspension to 10 though addition of $\mathrm{KOH}$. Three populations of pores are present in the foam microstructure: aligned, elongated pores (replicating ice dendrites), cracks in the copper walls (probably due to shrinkage during reduction) and micro-porosity within the copper walls (from incomplete sintering). The amount of micro-porosity is significantly reduced by increasing sintering time and temperature. Finally, density and porosity measurements have shown that total porosity of the sintered copper foams is about $80 \%$ for initial solids loading of $15 \mathrm{vol} \%$ in the slurry, and pore diameter is about $91 \mu \mathrm{m}$.

\section{Acknowledgments}

We thank C.A. Mirkin for the use of his lyophilizer, D.N. Seidman for the use of his tube furnace, and D. Isheim for helpful instructions about tube furnace operation.

\section{References}

1. Lefebvre, L.-P.; Banhart, J.; Dunand, D. Porous metals and metallic foams: Current status and recent developments. Adv. Eng. Mater. 2008, 10 (9), 775-787.

2. Conde, Y.; Despois, J.-F.; Goodall, R.; Marmottant, A.; Salvo, L; San Marchi, C; Mortensen, A. Replication processing of highly porous materials. Adv. Eng. Mater. 2006, 8 (9), 795-803.

3. Wang, Q.Z.; Cui, C.X.; Liu, S.J.; Zhao, L.C. Open-celled porous Cu prepared by replication of $\mathrm{NaCl}$ space-holders. Mater. Sci. Eng. A. 2010, 527 (4-5), 1275-1278.

4. Hakamada, M.; Asao, Y.; Kuromura, T.; Chen, Y.; Kusuda, H.; Mabuchi, M. Density dependence of the compressive properties of porous copper over a wide density range. Acta Mater. 2007, 55 (7), 2291-2299.

5. Zhang, E.; Wang, B. On the compressive behavior of sintered porous coppers with low to medium porosities-Part I: Experimental study. Int. J. Mech. Sci. 2005, 47 (4-5), 744-756.

6. Zhao, Y.Y.; Fung, T.; Zhang, L.P.; Zhang, F.L. Lost carbonate sintering process for manufacturing metal foams. Scripta Mater. 2005, 52 (4), 295-298.

7. Zhang, S.; Xing, Y.; Jiang, T.; Du, Z.; Li, F.; He, L.; Liu, W. A three-dimensional tin-coated nanoporous copper for lithium-ion battery anodes. J. Power Sources 2011, 196 (16), 6915-6919.

8. ERG Aerospace Corporation Homepage. Available online: http://www.ergaerospace.com (accessed on 1 July 2012).

9. Nakajima, H.; Hyun, S.K.; Ohashi, K.; Ota, K.; Murakami, K. Fabrication of porous copper by unidirectional solidification under hydrogen and its properties. Colloids Surf. A 2001, 179 (2-3), 209-214.

10. Hyun, S.K.; Murakami, K.; Nakajima, H. Anisotropic mechanical properties of porous copper fabricated by unidirectional solidification. Mater. Sci. Eng. A 2001, 299 (1-2), 241-248.

11. Zhang, H.; Cooper, A.I. Aligned porous structures by directional freezing. Adv. Mater. 2007, 19 (11), 1529-1533.

12. Deville, S.; Saiz, E.; Nalla, R.K.; Tomsia, A.P. Freezing as a path to build complex composites. Science 2006, 311 (5760), 515-518.

13. Deville, S.; Saiz, E.; Tomsia, A.P. Ice-templated porous alumina structures. Acta Mater. 2007, 55 (6), 1965-1974. 
14. Chino, Y.; Dunand, D.C. Directionally freeze-cast titanium foam with aligned, elongated pores. Acta Mater. 2008, 56 (1), 105-113.

15. Li, J.C.; Dunand, D.C. Mechanical properties of directionally freeze-cast titanium foams. Acta Mater. 2011, 59 (1), 146-158.

(C) 2012 by the authors; licensee MDPI, Basel, Switzerland. This article is an open access article distributed under the terms and conditions of the Creative Commons Attribution license (http://creativecommons.org/licenses/by/3.0/). 